\title{
Método Proximal para Problemas de Desigualdad Variacional: Caso no Monótono
}

\author{
Erik Papa ${ }^{1}$ \\ Lennin Ramire $z^{2}$ \\ (Recibido: 30/06/2015 - $\quad$ Aceptado: 23/02/2016)
}

Resumen: En el presente artículo introducimos un algoritmo de punto proximal inexacto usando distancias proximales para resolver el problema de desigualdad variacional cuando el operador involucrado en el modelo es pseudo-monótono y cuasi-monótono. Bajo algunas hipótesis naturales probamos que la sucesión generada por el método es convergente en el caso pseudo-monótono y débilmente convergente en el caso cuasi-monótono. Este enfoque extiende los resultados de Auslender, Teboulle y Ben-Tiba [1] y Brito et al.[3].

Palabras Claves: Problema de desigualdad variacional, distancia proximal, algoritmo proximal, operador cuasi-monótono, operador pseudo-monótono.

\section{Proximal Method for Variational Inequality Problems: Case no Monotone}

Abstract: In this paper we introduce an inexact proximal point algorithm using proximal distances for solving the variational inequality problem when the operator is pseudo-monotone and quasi-monotone. Under some natural assumptions we prove that the sequence generates by the method is convergent for the pseudo-monotone case and weakly convergent for the quasi-monotone one. This approach extends the result obtained by Auslender, Teboulle and Ben-Tiba [1] and Brito et al.[3].

Key Words: Variational inequality problem, proximal distance, proximal algorithm, quasimonotone operator, pseudo-monotone operator.

\section{Introducción}

El problema de desigualdad variacional (PDV) consiste en encontrar $x^{*} \in \bar{C}$ y $y^{*} \in T\left(x^{*}\right)$, tales que

$$
\left\langle y^{*}, x-x^{*}\right\rangle \geq 0 \text {, para cualquier } x \in \bar{C},
$$

donde $T$ es un operador punto conjunto de $I R^{n}$, esto es, una aplicación de $I R^{n}$ en el conjunto de partes de $I R^{n}, C$ es un conjunto convexo abierto de $I R^{n}$ y $\bar{C}$ es la clausura de $C$. El modelo anterior recubre como casos particulares a problemas de optimización, problemas de tráfico urbano, problemas de complementaridad lineal y no lineal, problemas de equilibrio económico, entre otros, ver por ejemplo Harker y Pang [5] y Facchinei y Pang [4]. Existen diversos métodos para resolver problemas de desigualdad variacional, por ejemplo, los métodos basados en funciones de mérito, métodos de punto interior, métodos proyectivos, métodos de punto proximal, métodos splitting, entre otros. Por la importancia y las propiedades de convergencia global del método

\footnotetext{
${ }^{1}$ UNMSM, Facultad de Ciencias Matemáticas, e-mail: erikpapa@gmail.com

${ }^{2}$ UNMSM, Facultad de Ciencias Matemáticas, e-mail: lenninmr@gmail.com
} 
de punto proximal en este artículo estamos interesados en resolver el problema (1) usando este método pero cuando el operador no es necesariamente monótono. Recordemos que en el caso monótono es posible obtener la convergencia global del método a una solución de (1), ver por ejemplo Teorema 12.3.7 de Facchinei y Pang ver [4].

Especificamente, estudiamos las iteraciones proximales inexactas de la forma: dado $x^{k-1} \in C$ encontrar $x^{k} \in C$ y $u^{k} \in T\left(x^{k}\right)$ tales que

$$
u^{k}+\lambda_{k} \nabla_{1} d\left(x^{k}, x^{k-1}\right)=e^{k}
$$

donde $T$ es un operador pseudo-monótono o cuasi-monótono, $\lambda_{k}$ es un parámetro positivo, $d$ es la distancia proximal, ver Subsección 3.1, y $e^{k} \in I R^{n}$ es el error de aproximación dado por:

$$
\begin{gathered}
\sum_{k=1}^{+\infty} \frac{\left\|e^{k}\right\|}{\lambda_{k}}<+\infty \\
\sum_{k=1}^{+\infty} \frac{\left|\left\langle e^{k}, x^{k}\right\rangle\right|}{\lambda_{k}}<+\infty
\end{gathered}
$$

El aporte principal de este trabajo es la demostración de convergencia del método cuando $T$ es un operador pseudo-monótono y la convergencia débil cuando el operador es cuasi-monótono. Nuestros resultados extienden la convergencia, para el caso no monótono y caso inexacto, de los métodos introducidos por Auslender, Teboulle and Ben-Tiba [1] y Brito et al. [3].

El artículo está organizado de la siguiente manera: En la Sección 2 presentamos la metodología empleada en el estudio del trabajo de investigación. En la Sección 3 mostramos los resultados básicos empleados a lo largo del artículo. En la Sección 3.2 introducimos el método proximal y en la Sección 3.3 estudiamos la convergencia de la sucesión generada por el método, analisando el caso pseudo-monótono y el caso cuasi-monótono.

\section{Metodología}

La metodología de trabajo fue dividida en las siguientes partes:

\section{$2.1 \quad$ El universo}

El universo de nuestro trabajo de investigación son los problemas de desigualdad variacional (PDV) en el espacio euclidiano.

\subsection{Técnicas de recopilación de datos}

Para el desarrollo de la investigación fue necesario la búsqueda de información en revistas científicas indexadas, uso de bibliotecas y las revistas que no fueron de fácil acceso se pudieron conseguir gracias a personas conocidas en otras universidades, tales como la Universidad de Chile y la Pontificia Universidad Católica de Chile.

\subsection{Técnicas descriptivas para la contrastación o demostración de las hipó- tesis}

La metodología usada fue del tipo inductivo-deductivo de los resultados de recientes investigaciones, con el objetivo de poderlos adaptar a nuestro caso y que nos llevó a la obtención de los resultados deseados para nuestra investigación. 


\section{Resultados y Discusión}

A lo largo de este trabajo $I R^{n}$ es el espacio euclideano dotado con el producto interno canónico $\langle$,$\rangle y la norma en x$ dada por $\|x\|:=\langle x, x\rangle^{1 / 2}$.

Lema 3.1. Sea $\left\{v_{k}\right\},\left\{\gamma_{k}\right\}, y\left\{\beta_{k}\right\}$ sucesiones no negativas de números reales que satisfacen $v_{k+1} \leq$ $\left(1+\gamma_{k}\right) v_{k}+\beta_{k}$ y tal que $\sum_{k=1}^{\infty} \beta_{k}<\infty, \sum_{k=1}^{\infty} \gamma_{k}<\infty$. Entonces, la sucesión $\left\{v_{k}\right\}$ converge.

Prueba. Ver Lema 2, pp. 44, de Polyak [7].

Definición 3.2. Sea $T: I R^{n} \rightrightarrows I R^{n}$ un operador punto conjunto. El dominio y el gráfico de $T$ están definidos como

$$
\begin{gathered}
D(T)=\left\{x \in I^{n}: T(x) \neq \emptyset\right\} . \\
G(T)=\left\{(x, v) \in I R^{n} \times I R^{n}: x \in D(T), v \in T(x)\right\} .
\end{gathered}
$$

Definición 3.3. Un operador punto conjunto $T: I R^{n} \rightrightarrows I R^{n}$ es cerrado en $\bar{x}$ si, y solo si, para cualquier sucesión $\left\{x^{k}\right\} \subset I^{n}$ y cualquier sucesión $\left\{v^{k}\right\} \subset \operatorname{IR}^{n}$ tales que $\left(x^{k}, v^{k}\right) \in G(T)$ y $\left(x^{k}, v^{k}\right) \rightarrow(\bar{x}, \bar{v})$, se tiene que $\bar{v} \in T(\bar{x})$.

Proposición 3.4. Un operador $T: I R^{n} \rightrightarrows I R^{n}$ es localmente acotado si, y sólo si, $T(B)$ es acotado para algún conjunto acotado B. Equivalentemente, cada vez que $v^{k} \in T\left(x^{k}\right)$ y la suceción $\left\{x^{k}\right\} \subset I R^{n}$ es acotada, entonces la sucesión $\left\{v^{k}\right\}$ es acotada.

Prueba. Ver Proposición 5.15 de Rockafellar y Wets [8].

Definición 3.5. Un operador $T: I R^{n} \rightrightarrows I R^{n}$ es:

i. Fuertemente monótono si existe una constante $\alpha>0$ tal que

$$
\langle u-v, x-y\rangle \geq \alpha\|x-y\|^{2} .
$$

para cualesquiera $(x, u),(y, v) \in G(T)$.

ii. Monótono si

$$
\langle u-v, x-y\rangle \geq 0,
$$

para cualesquiera $(x, u),(y, v) \in G(T)$.

iii. Pseudo-monótono si

$$
\langle v, x-y\rangle \geq 0 \text { implica }\langle u, x-y\rangle \geq 0,
$$

para cualesquiera $(x, u),(y, v) \in G(T)$.

iv. Cuasi-monótono si

$$
\langle v, x-y\rangle>0 \text { implica }\langle u, x-y\rangle \geq 0 .
$$

para cualesquiera $(x, u),(y, v) \in G(T)$.

v. Débilmente monótono si existe una constante $\rho>0$ tal que

$$
\langle u-v, x-y\rangle \geq-\rho\|x-y\|^{2} .
$$

para cualesquiera $(x, u),(y, v) \in G(T)$. 


\subsection{Distancia proximal y distancia proximal inducida}

En esta subsección presentamos la definición de distancia proximal y distancia proximal inducida, introducida por Auslender y Teboulle [2].

Definición 3.6. Una función $d: I R^{n} \times I R^{n} \rightarrow I R_{+} \cup\{+\infty\}$ es denominada distancia proximal en un conjunto convexo y abierto $C$ si para cada $y \in C$ se satisface las siguientes propiedades:

i. $d(\cdot, y)$ es propia, semicontinua inferior, estrictamente convexa y continuamente diferenciable en $C$;

ii. $\operatorname{dom} d(\cdot, y) \subset \bar{C}$ y dom $\partial_{1} d(\cdot, y)=C$, donde $\partial_{1} d(\cdot, y)$ denota el clásico subdiferencial de la función $d(\cdot, y)$ con respecto a la primera variable;

iii. $d(\cdot, y)$ es coerciva en $\operatorname{IR}^{n}$ (es decir, $\left.\operatorname{lím}_{\|u\| \rightarrow \infty} d(u, y)=+\infty\right)$.

iv. $d(y, y)=0$.

Denotamos por $D(C)$ a la familia de funciones satisfaciendo esta definición.

La propiedad i. es necesaria para preservar la convexidad de $d(\cdot, y)$, la propiedad ii fuerza que la iteración del método proximal permanezca en $C$, y la propiedad iii es útil para garantizar las existencias de las iteraciones proximales del método propuesto. Para cada $y \in C, \nabla_{1} d(\cdot, y)$ denota el gradiente de la función $d(\cdot, y)$ con respecto a la primera variable. Notemos que por definición $d(\cdot, \cdot) \geq 0$ y de iv. el mínimo global de $d(\cdot, y)$ se obtiene en $y$, y así tenemos que $\nabla_{1} d(y, y)=0$.

Definición 3.7. Dado $d \in D(C)$, una función $H: I R^{n} \times I R^{n} \rightarrow I R_{+} \cup\{+\infty\}$ es denominada distancia proximal inducida por $d$ si $H$ tiene valores finitos en $C \times C$ y para cualesquiera $a, b \in C$ se satisface:

(Ii) $H(a, a)=0$.

(Iii) $\left\langle c-b, \nabla_{1} d(b, a)\right\rangle \leq H(c, a)-H(c, b), \quad \forall c \in C$.

Denotaremos $(d, H) \in \mathcal{F}(C)$ a la distancia proximal y distancia proximal inducida que satisfacen las premisas de la Definición 3.7.

Denotamos por $(d, H) \in \mathcal{F}(\bar{C})$ si existe una función $H$ tal que:

(Iiii) $H$ tiene valores finitos en $\bar{C} \times C$ satisfaciendo (Ii) y (Iii), para cada $c \in \bar{C}$.

(Iiv) Para cada $c \in \bar{C}, H(c, \cdot)$ tiene conjuntos de nivel acotados en $C$.

Finalmente, denotaremos $(d, H) \in \mathcal{F}_{+}(\bar{C})$ si

(Iv) $(d, H) \in \mathcal{F}(\bar{C})$.

(Ivi) Para cualesquiera $y \in \bar{C}$ y $\left\{y^{k}\right\} \subset C$ acotada con $\operatorname{lím}_{k \rightarrow+\infty} H\left(y, y^{k}\right)=0$, entonces $\lim _{k \rightarrow+\infty} y^{k}=y$.

(Ivii) Para cualesquiera $y \in \bar{C}$ y $\left\{y^{k}\right\} \subset C$ tal que $\operatorname{lím}_{k \rightarrow+\infty} y^{k}=y$, entonces $\operatorname{lím}_{k \rightarrow+\infty} H\left(y, y^{k}\right)=$ 0 .

El resultado principal del método propuesto será cuando $(d, H) \in \mathcal{F}_{+}(\bar{C})$. Varios ejemplos de distancias proximales que se ajustan a las definiciones anteriores, por ejemplo distancias Bregman, distancias $\phi$ - divergencias, distancias homogéneas de segundo orden, fueron dadas por Auslender y Teboulle ver [2]. 
Definición 3.8. Sea $(d, H) \in \mathcal{F}(\bar{C})$. Una sucesión $\left\{z^{l}\right\} \subset C$ es llamada $H$-cuasi-Fejér convergente en $U \subset \bar{C}$ si para todo $u \in U$ existe una sucesión $\left\{\epsilon_{l}\right\}$, con $\epsilon_{l} \geq 0$ y $\sum_{l=1}^{+\infty} \epsilon_{l}<+\infty$ tal que para cada l se cumple

$$
H\left(u, z^{l}\right) \leq H\left(u, z^{l-1}\right)+\epsilon_{l}
$$

Proposición 3.9. Sea $(d, H) \in \mathcal{F}_{+}(\bar{C})$ y $\left\{z^{l}\right\} \subset C$ una sucesión $H$-cuasi-Fejér convergente en $U \subset \bar{C}$ entonces $\left\{z^{l}\right\}$ es acotada y si existe un punto de acumulación $\bar{z} \in U$, entonces toda la sucesión $\left\{z^{l}\right\}$ converge a $\bar{z}$.

Prueba. Sea $u \in U$, de la hipótesis de $H$-cuasi-Fejér convergencia tenemos que

$$
H\left(u, z^{l}\right) \leq H\left(u, z^{0}\right)+\sum_{l=1}^{+\infty} \epsilon_{l} .
$$

Así, $z^{l} \in L_{H}(u, \alpha)=\{y \in C: H(u, y) \leq \alpha\}$, donde $\alpha=H\left(u, z^{0}\right)+\sum_{l=1}^{+\infty} \epsilon_{l}$. De la Definición 3.7, Iiv, $L_{H}(u, \alpha)$ es acotado y por lo tanto $\left\{z^{l}\right\}$ es una sucesión acotada.

Sean $\bar{z}$ y $z^{*}$ dos puntos de acumulación de $\left\{z^{l}\right\}$ donde $z^{l_{j}} \rightarrow \bar{z}$ y $z^{l_{k}} \rightarrow z^{*}$ con $\bar{z} \in U$, entonces de la Definición 3.7 Ivii, $H\left(\bar{z}, z^{l_{j}}\right) \rightarrow 0$ y $H\left(z^{*}, z^{l_{k}}\right) \rightarrow 0$. Como $H\left(\bar{z}, z^{l}\right)$ es convergente, ver Lema 3.1, y la subsucesión $H\left(\bar{z}, z^{l_{j}}\right)$ converge a cero se tiene que $H\left(\bar{z}, z^{l}\right) \rightarrow 0$ y en particular $H\left(\bar{z}, z^{l_{k}}\right) \rightarrow 0$. Usando la Definición 3.7, Ivi, obtenemos que $z^{l_{k}} \rightarrow \bar{z}$ y por la unicidad del límite se tiene que $z^{*}=\bar{z}$. Así, $\left\{z^{l}\right\}$ converge a $\bar{z}$.

(Iviii) Dada una matriz de $B \in I R^{n \times n}$ simétrica definida positiva y $d \in \mathcal{D}(C)$. Decimos que $d$ es fuertemente convexa con respecto a la primera variable y con respecto a la norma $\|.\|_{B}$, si para cada $y \in C$ existe $\alpha>0$ tal que

$$
\left\langle\nabla_{1} d\left(x_{1}, y\right)-\nabla_{1} d\left(x_{2}, y\right), x_{1}-x_{2}\right\rangle \geq \alpha\left\|x_{1}-x_{2}\right\|_{B}^{2},
$$

para cualesquiera $x_{1}, x_{2} \in C$.

Algunos ejemplos de distancias proximales que satisfacen la definición anterior son las distancias homogéneas de segundo orden, ver Brito et al. [3], Lema 3.1.

Lema 3.10. Sea $B \in I R^{n \times n}$ una matriz simétrica definida positiva. Fijamos $y \in C$, si $T$ es un operador débilmente monótono con constante $\rho>0, d(., y)$ es fuertemente convexa en $C$ con respecto a la norma $\left\|_{1 .}\right\|_{B}$, con constante $\alpha$ y $\left\{\beta_{k}\right\}$ es una sucesión de números positivos que satisfacen

$$
\beta_{k} \geq \beta>\frac{\rho}{\alpha \lambda_{\min }(B)},
$$

donde $\lambda_{\text {min }}(B)$ denota el menor autovalor de $B$, entonces el operador $F()=.T()+.\beta_{k} \nabla_{1} d(., y)$ es fuertemente monótono en $C$ con constante $\beta \alpha \lambda_{\text {min }}(B)-\rho$.

Prueba. Similar al Lema 5.1 de Brito, et al. [3].

\subsection{Método proximal inexacto}

Estamos interesados en resolver el (PDV): encontrar $x^{*} \in \bar{C}, y^{*} \in T\left(x^{*}\right)$, tales que

$$
\left\langle y^{*}, x-x^{*}\right\rangle \geq 0, \forall x \in \bar{C},
$$


donde $T$ es un operador punto conjunto de $I R^{n}$ no necesariamente monótono, $C$ es un conjunto no vacio convexo y abierto, $\bar{C}$ es la clausura de $C$ en $I R^{n}$ y

$$
D(T) \cap C \neq \emptyset .
$$

A seguir proponemos una extensión del método de punto proximal con una distancia proximal para resolver el problema planteado.

\section{Algoritmo inexacto}

Inicialización: Sea $\left\{\lambda_{k}\right\}$ una sucesión de parámetros positivos y un punto inicial:

$$
x^{0} \in C .
$$

Paso Principal: Para cada $k=1,2, \ldots$, y $x^{k-1} \in C$, encontrar $x^{k} \in C$ y $u^{k} \in T\left(x^{k}\right)$, tales que:

$$
u^{k}+\lambda_{k} \nabla_{1} d\left(x^{k}, x^{k-1}\right)=e^{k}
$$

donde $d$ es una distancia proximal tal que $(d, H) \in F_{+}(\bar{C})$ y $e^{k}$ es un error de aproximación.

Criterio de Parada: Si $x^{k}=x^{k-1}$, entonces finalizar. Caso contrario, hacer $k-1 \leftarrow k$ y retornar al paso principal.

En todo este artículo, asumiremos las siguientes hipótesis:

(H1) Las iteraciones $\left\{x^{k}\right\}$ existen y pertenecen a $C$, esto es, para todo $k$, existe $x^{k} \in C$.

(H2) El conjunto solución $\operatorname{SOL}(T, \bar{C})$ del $(\mathrm{PDV})$ es no vacío.

Observación 3.11. Algunas condiciones suficientes para que se cumpla la hipótesis (H1) fueron presentados en los teoremas 5.1 y 5.2 de Brito et al. ver [3].

\subsection{Análisis de convergencia}

En esta sección, bajo algunas condiciones naturales, probaremos que el método propuesto converge. Dividiremos el análisis en dos casos, el caso pseudo-monótono y el caso cuasi-monótono. Además, como estamos interesados en la convergencia asintótica del método, también asumimos que en cada iteración $x^{k} \neq x^{k-1}$ para todo $k \in I N$. En efecto, si $x^{k}=x^{k-1}$, entonces $\nabla_{1} d\left(x^{k}, x^{k-1}\right)=0$ y de (10)-(11) se tiene que $e^{k} \in T\left(x^{k}\right)$, esto es, $x^{k}$ es una solución aproximada del (PDV).

\subsection{Caso pseudo-monótono}

Proposición 3.12. Sea $T$ un operador pseudo-monótono, $(d, H) \in \mathcal{F}(\bar{C})$, y supongamos que se cumplen las hipótesis (H1) y (H2). Entonces :

$$
H\left(\bar{x}, x^{k}\right) \leq H\left(\bar{x}, x^{k-1}\right)-\frac{1}{\lambda_{k}}\left\langle e^{k}, \bar{x}-x^{k}\right\rangle,
$$

para todo $\bar{x} \in \operatorname{SOL}(T, \bar{C})$.

Prueba. Dado $\bar{x} \in \operatorname{SOL}(T, \bar{C})$, tenemos que $\langle\bar{u}, z-\bar{x}\rangle \geq 0$ para todo $z \in \bar{C}$ y todo $\bar{u} \in T(\bar{x})$, en particular $\left\langle\bar{u}, x^{k}-\bar{x}\right\rangle \geq 0$. Usando la pseudo-monotonicidad de $T$, tenemos que para todo 
$u^{k} \in T\left(x^{k}\right)$ se tiene $\left\langle u^{k}, x^{k}-\bar{x}\right\rangle \geq 0$. Ahora de (11) tenemos:

$0 \leq\left\langle u^{k}, x^{k}-\bar{x}\right\rangle=\left\langle e^{k}-\lambda_{k} \nabla_{1} d\left(x^{k}, x^{k-1}\right), x^{k}-\bar{x}\right\rangle=\left\langle e^{k}, x^{k}-\bar{x}\right\rangle+\lambda_{k}\left\langle\nabla_{1} d\left(x^{k}, x^{k-1}\right), \bar{x}-x^{k}\right\rangle$, esto es,

$$
0 \leq\left\langle e^{k}, x^{k}-\bar{x}\right\rangle+\lambda_{k}\left\langle\nabla_{1} d\left(x^{k}, x^{k-1}\right), \bar{x}-x^{k}\right\rangle .
$$

Como $(d, H) \in \mathcal{F}(\bar{C})$ y de la Definición 3.7, Iii, se concluye que

$$
0 \leq\left\langle e^{k}, x^{k}-\bar{x}\right\rangle+\lambda_{k}\left[H\left(\bar{x}, x^{k-1}\right)-H\left(\bar{x}, x^{k}\right)\right] .
$$

Luego

$$
H\left(\bar{x}, x^{k}\right) \leq H\left(\bar{x}, x^{k-1}\right)-\frac{1}{\lambda_{k}}\left\langle e^{k}, \bar{x}-x^{k}\right\rangle .
$$

Proposición 3.13. Sea $T$ un operador pseudo-monótono, $(d, H) \in \mathcal{F}(\bar{C})$, y supongamos que se cumplen las hipótesis $\mathbf{( H 1 )}$ y (H2). Si las condiciones adicionales siguientes se satisfazen:

$$
\begin{gathered}
\sum_{k=1}^{+\infty} \frac{\left\|e^{k}\right\|}{\lambda_{k}}<+\infty \\
\sum_{k=1}^{+\infty} \frac{\mid\left\langle e^{k}, x^{k}||\right.}{\lambda_{k}}<+\infty
\end{gathered}
$$

entonces

a. $\left\{x^{k}\right\}$ es $H$-cuasi-Féjer convergente en $\operatorname{SOL}(T, \bar{C})$. esto es,

$$
H\left(\bar{x}, x^{k}\right) \leq H\left(\bar{x}, x^{k-1}\right)+\epsilon^{k},
$$

para todo $\bar{x} \in \operatorname{SOL}(T, \bar{C})$, donde $\epsilon^{k}=\frac{1}{\lambda_{k}}\left(\left\|e^{k}\right\|\|\bar{x}\|+\left|\left\langle e^{k}, x^{k}\right\rangle\right|\right) \operatorname{con} \sum_{k=1}^{+\infty} \epsilon^{k}<+\infty$.

b. $\left\{H\left(\bar{x}, x^{k}\right)\right\}$ convergente para todo $\bar{x} \in \operatorname{SOL}(T, \bar{C})$.

c. $\left\{x^{k}\right\}$ es acotada.

\section{Prueba.}

a. Usando la desigualdad de Cauchy-Schwarz en (12) tenemos para todo $\bar{x} \in \operatorname{SOL}(T, \bar{C})$ que:

$$
H\left(\bar{x}, x^{k}\right) \leq H\left(\bar{x}, x^{k-1}\right)+\frac{1}{\lambda_{k}}\left(\left\|e^{k}\right\|\|\bar{x}\|+\left|\left\langle e^{k}, x^{k}\right\rangle\right|\right)
$$

Sea $\epsilon^{k}=\frac{1}{\lambda_{k}}\left(\left\|e^{k}\right\|\|\bar{x}\|+\left|\left\langle e^{k}, x^{k}\right\rangle\right|\right)$, entonces $H\left(\bar{x}, x^{k}\right) \leq H\left(\bar{x}, x^{k-1}\right)+\epsilon^{k}$, y de las hipótesis (13) y (14) tenemos que $\sum_{k=1}^{+\infty} \epsilon^{k}<\infty$.

b. Es inmediato de a. y Lema 3.1.

c. Inmediato también de a. y de la Proposición 3.9.

Ahora demostraremos que el algoritmo propuesto resuelve el (PDV) donde $T$ es un operador pseudo-monótono. Nosotros necesitaremos la siguientes hipótesis adicional 
(H3) $T$ es un operador localmente acotado y $G(T)$ es cerrado.

La prueba del siguiente teorema es similar al Teorema 9 de Langenberg y Tichatschke ver [6].

Teorema 3.14. Supongamos que $T$ un operador pseudo-monótono y que se cumplen las hipótesis (H1)-(H3). Si $(d, H) \in \mathcal{F}_{+}(\bar{C}), 0<\lambda_{k}<\bar{\lambda}$, para algún $\bar{\lambda}>0$, entonces $\left\{x^{k}\right\}$ converge a un elemento de $\operatorname{SOL}(T, \bar{C})$.

Demostración. Sea $x^{*}$ un punto de acumulación de $\left\{x^{k}\right\}$ y $\left\{x^{k_{j}}\right\}$ una subsucesión que converge para $x^{*}$, de (11) se tiene que para cada $x \in \bar{C}$ :

$$
\left\langle u^{k}, x-x^{k}\right\rangle=\left\langle e^{k}, x-x^{k}\right\rangle-\lambda_{k}\left\langle\nabla_{1} d\left(x^{k}, x^{k-1}\right), x-x^{k}\right\rangle .
$$

En vista de (13) y que $\left\{\lambda_{k}\right\}$ es acotada superiormente se tiene que $\left\|e^{k}\right\| \rightarrow 0$. Luego, como $\left\{x^{k}\right\}$ es acotada, obtenemos que $\left\langle e^{k}, x-x^{k}\right\rangle \rightarrow 0$, luego, solamente será necesario analizar la convergencia de

$$
-\lambda_{k}\left\langle\nabla_{1} d\left(x^{k}, x^{k-1}\right), x-x^{k}\right\rangle \text {. }
$$

De la Definición 3.7 (Iii), tenemos que

$$
-\lambda_{k}\left\langle\nabla_{1} d\left(x^{k}, x^{k-1}\right), x-x^{k}\right\rangle \geq \lambda_{k}\left[H\left(x, x^{k}\right)-H\left(x, x^{k-1}\right)\right] .
$$

Analizaremos dos casos:

Si $H\left(x, x^{k}\right)$ converge, entonces $\lambda_{k}\left[H\left(x, x^{k}\right)-H\left(x, x^{k-1}\right)\right] \rightarrow 0$, dado que $\left\{\lambda_{k}\right\}$ es acotada, y así:

$$
\liminf _{k \rightarrow \infty}\left\langle u^{k}, x-x^{k}\right\rangle \geq 0 \text {. }
$$

Si $H\left(x, x^{k}\right)$ no es convergente, entonces la sucesión no es monótonamente decreciente y por lo tanto hay una infinidad de $j \in I N$ tales que $H\left(x, x^{j}\right) \geq H\left(x, x^{j-1}\right)$. Sea $\left\{k_{j}\right\} \subset I N$ una subsucesión con $H\left(x, x^{k_{j}}\right) \geq H\left(x, x^{k_{j}-1}\right)$, para todo $j \in I N$, entonces

$$
\liminf _{j \rightarrow \infty}\left\langle u^{k_{j}}, x-x^{k_{j}}\right\rangle \geq \lambda_{k_{j}}\left[H\left(x, x^{k_{j}}\right)-H\left(x, x^{k_{j}-1}\right)\right] \geq 0,
$$

y así:

$$
\liminf _{j \rightarrow \infty}\left\langle u^{k_{j}}, x-x^{k_{j}}\right\rangle \geq 0
$$

para cada $x \in \bar{C}, u^{k_{j}} \in T\left(x^{k_{j}}\right)$.

Dado que $T$ es localmente acotado y como $\left\{x^{k}\right\}$ es acotado entonces por la Proposición 3.4, la sucesión $\left\{u^{k}\right\}$ es acotada. Luego, existe una subsucesión de $\left\{u^{k_{l}}\right\}$ que converge para algún $u^{*}$. Como $G(T)$ es cerrado se tiene que $u^{*} \in T\left(x^{*}\right)$. Podemos suponer sin pérdida de generalidad que $u^{k_{j}} \rightarrow u^{*} \in T\left(x^{*}\right)$. En consecuencia $\left\langle u^{*}, x-x^{*}\right\rangle \geq 0$ para todo $x \in \bar{C}$ y $u^{*} \in T\left(x^{*}\right)$, implica que $x^{*} \in \operatorname{SOL}(T, \bar{C})$.

Usando la Proposición 3.9, tenemos que $\left\{x^{k}\right\}$ converge para $x^{*}$.

\subsection{Caso cuasi-monótono}

Imitando los pasos del trabajo de Brito et al. (2012), quienes trabajaron para una distancia específica, asumiremos que $T$ es un operador cuasi-monótono y consideremos el siguiente subconjunto de $\operatorname{SOL}(T, \bar{C})$ :

$$
\operatorname{SOL}^{*}(T, \bar{C})=\left\{x^{*} \in \operatorname{SOL}(T, \bar{C}): \exists u^{*} \neq 0, u^{*} \in T\left(x^{*}\right)\right\} .
$$


Para esta subsección consideramos $\operatorname{SOL}(T, \bar{C}) \cap \partial \bar{C} \neq \varnothing$, donde $\partial \bar{C}$ denota la frontera de $\bar{C}$. De esta forma usaremos la siguiente hipótesis:

(H2)' $\operatorname{SOL}^{*}(T, \bar{C}) \neq \varnothing$

El siguiente lema fue probado por Brito et al. ver [3] y Langenberg ver [6], pero por cuestión de completitud establecemos la prueba.

Lema 3.15. Asumamos que la hipótesis (H2)' es satisfecha. Si $x^{*} \in \operatorname{SOL}^{*}(T, \bar{C})$, entonces

$$
\left\langle u^{*}, w-x^{*}\right\rangle>0 \text {, para cualquier } w \in C,
$$

donde $u^{*} \neq 0, u^{*} \in T\left(x^{*}\right)$.

Prueba. Dado $x^{*} \in S O L^{*}(T, \bar{C})$, entonces $\left\langle u^{*}, x-x^{*}\right\rangle \geq 0$ para todo $x \in \bar{C}$. Supongamos que existe $\bar{w} \in C$ tal que $\left\langle u^{*}, \bar{w}-x^{*}\right\rangle=0$. Desde que $\bar{w} \in C$, existe $B(\bar{w}, r) \subset C$, donde $B(\bar{w}, r)$ es una bola centrada en $\bar{w}$ y de radio $r>0$.

Ahora como $\left\|u^{*}\right\|>0$ y $r>0$, existe $\epsilon>0\left(\epsilon<r\left\|u^{*}\right\|\right)$ tal que $\bar{x}=\bar{w}-\frac{\epsilon}{\left\|u^{*}\right\|^{2}} u^{*} \in B(\bar{w}, r)$ y por lo tanto $\bar{x} \in C$. Consecuentemente $\left\langle u^{*}, \bar{x}-x^{*}\right\rangle=-\epsilon<0$, lo que es una contradicción.

Proposición 3.16. Sea $T$ un operador cuasi-monótono, $(d, H) \in \mathcal{F}(\bar{C})$, y supongamos que se cumplen las hipótesis $\mathbf{( H 1 )}$ y (H2)'. Entonces :

$$
H\left(\bar{x}, x^{k}\right) \leq H\left(\bar{x}, x^{k-1}\right)-\frac{1}{\lambda_{k}}\left\langle e^{k}, \bar{x}-x^{k}\right\rangle,
$$

para todo $\bar{x} \in S O L^{*}(T, \bar{C})$.

Prueba. Dado $\bar{x} \in \operatorname{SOL}^{*}(T, \bar{C})$, tenemos que $\langle\bar{u}, x-\bar{x}\rangle \geq 0$ para todo $x \in \bar{C}$ y todo $\bar{u} \in T(\bar{x})$ con $\bar{u} \neq 0$. Por la hipótesis (H1), $x^{k} \in C$ y usando el Lema 3.15 tenemos que $\left\langle\bar{u}, x^{k}-\bar{x}\right\rangle>0$. Usando la propiedad de que $T$ es cuasi-monótono, tenemos que para todo $u^{k} \in T\left(x^{k}\right)$ se tiene $\left\langle u^{k}, x^{k}-\bar{x}\right\rangle \geq 0$, a partir de aquí, los pasos son los mismos que la demostración de la Proposición 3.12 .

Proposición 3.17. Sea $T$ un operador cuasi-monótono, $(d, H) \in \mathcal{F}(\bar{C})$, y supongamos que se cumplen las hipótesis (H1) y (H2)'. Si las condiciones de error (13) y (14) se satisfacen, entonces

a. $\left\{x^{k}\right\}$ es $H$-cuasi-Féjer convergente en $\operatorname{SOL}^{*}(T, \bar{C})$.

b. $\left\{H\left(\bar{x}, x^{k}\right)\right\}$ convergente para todo $\bar{x} \in S O L^{*}(T, \bar{C})$.

c. $\left\{x^{k}\right\}$ es acotada.

Demostración. Similar a la prueba de la Proposición 3.13.

Denotemos Acc $\left(x^{k}\right)$ como el conjunto de todos los puntos de acumulación de $\left\{x^{k}\right\}$.

Teorema 3.18. Supongamos que $T$ es un operador cuasi-monótono y que se cumplen las hipótesis (H1), (H2)' y (H3). Si $(d, H) \in \mathcal{F}_{+}(\bar{C}), 0<\lambda_{k}<\bar{\lambda}$, para algún $\bar{\lambda}>0$, entonces

(i) $\left\{x^{k}\right\}$ converge debilmente a un elemento de $\operatorname{SOL}(T, \bar{C})$, esto es, Acc $\left(x^{k}\right) \neq \varnothing$ y todo elemento de Acc $\left(x^{k}\right)$ es una solución de $\operatorname{VI}(T, \bar{C})$.

(ii) Si Acc $\left(x^{k}\right) \cap S O L^{*}(T, C) \neq \varnothing$ entonces $\left\{x^{k}\right\}$ converge para un elemento de la solución $\operatorname{SOL}^{*}(T, C)$.

Demostración. Similar a la demostración del Teorema 3.14. 


\subsection{Discusiones}

Este artículo muestra avances importantes en el método del punto proximal inexacto para resolver problemas de desigualdad variacional cuando el operador involucrado en el modelo no es necesariamente monótono.

Probamos la convergencia global en el caso pseudo-monótono y convergencia débil en el caso cuasi-monótono. Una hipótesis fuerte asumida en todo el trabajo es que las iteraciones dadas por el método existen y pertenecen al interior del conjunto convexo del modelo, creemos que en futuros trabajos se debe encontrar hipótesis apropiadas para garantizar la existencia de estas iteraciones.

La convergencia fuerte del método proximal para el caso cuasi-monótono fue obtenida por Langenberg ver [6] usando distancias de Bregman y asumiendo ciertas hipótesis razonables, siguiendo sus ideas tal vez se pueda extender esta convergencia para cualquier distancia proximal.

\section{Conclusiones}

- Se presenta algunos resultados de convergencia fuerte y débil del algoritmo de punto proximal inexacto con distancias proximales para resolver el problema de desigualdad variacional, restricto a un conjunto convexo, cuando el operador no es necesariamente monótono. Se espera en futuros trabajos dar condiciones de convergencia para el operador cuasimonótono, así como también su implementación computacional y encontrar relacionescaraterizaciones entre el operador cuasi-monótono y débilmente monótono.

- Este trabajo motiva investigar la siguiente pregunta: ¿Es viable desarrollar un algoritmo forward-backward inexacto con distancias proximales para resolver el (PDV)?

- En un futuro trabajo se pretende introducir un algoritmo de punto proximal inexacto usando distancias proximales, para resolver problemas de equilibrio y problemas de desigualdad variacional mixto.

\section{Agradecimientos}

Los autores agradecemos al Instituto de Investigación de la Facultad de Ciencias Matemáticas (UNMSM) por la oportunidad brindada para someter el presente artículo.

Los autores también agradecemos a Tran Nguyen Bao del Centro de Modelamiento Matemático (CMM) de la Universidad de Chile y a Gredy Salmerón de la Pontificia Universidad Católica de Chile por sus comentarios y facilitar las revistas científicas.

Finalmente, la investigación del primer autor fue financiada por el Proyecto Pos Doctoral CAPESFAPERJ Edital PAPD-2011. 


\section{Referencias Bibliográficas}

[1] Auslender, A., Teboulle, M., Ben-Tiba, S. (1999). Interior proximal and multiplier methods based on second order homogeneous functionals. MATH OPER. RESEARCH. Vol 24, $\mathrm{N}^{\circ} 3$ : 645-668.

[2] Auslender, A., Teboulle, M. (2006). Interior gradient and proximal methods for convex and conic optimization. SIAM J. OPTIM. Vol 16, $\mathrm{N}^{\circ}$ 3: 697-725.

[3] Brito, S., da Cruz Neto, J. X., Lopes J. O. (2012). Interior algorithm for quasiconvex programming problems and variational inequalities with linear constraints. J. OPTIM THEORY APPL. Vol 154, N 1: 217-234.

[4] Facchinei, F., Pang, J. S. (2003). Finite-dimensional variational inequalities and complementarity problems. Vol I and II, Springer Series in Operations Research. Springer-Verlag, New York.

[5] Harker, P. T., Pang, J. S. (1990). Finite-dimensional variational inequality and nonlinear complementarity problems: a survey of theory, algorithms and applications. MATH. PROGRAM., Vol 48:161-220.

[6] Langenberg, N., Tichatschke, R. (2012). Interior proximal methods for quasiconvex optimization. J. GLOB OPTIM. Vol 52: 641-661.

[7] Polyak, B. T. (1987). Introduction to optimization, optimization software. Optimization Software, New York.

[8] Rockafellar, R. T., Wets, R. (1998). Variational analysis. Grundlehren der Mathematischen, Springer. 